\title{
Mitochondrial internal structure correlated with respiratory activity in cultured human cells.
}

\author{
J. M. L. Selker, K. Snyder, R. W. Gilkerson, R. Rossignol and R. A. Capaldi \\ Institute of Molecular Biology, University of Oregon, Eugene, OR 97403
}

Three morphologically distinguishable membranes exist in the mitochondrion: 1) outer membrane, 2) inner boundary membrane, parallel to the outer membrane, and 3) cristal membrane. The outer membrane contains numerous pores that allow small molecules to move across, whereas the inner boundary membrane and the cristal membrane are not permeant and contain the respiratory chain.

We have investigated two situations in which the ratio of cristal membrane to inner boundary membrane becomes dramatically altered. Respiratory-deficient rho zero MRC- 5 cells (human lung fibroblasts), totally lacking mitochondrial DNA, have fewer cristae than wild type MRC-5 cells (Fig. 1). The ratio of cristal membrane to inner boundary membrane is 2 for wild type and 1 for rho zero cells [1]. The organization of cristal membranes is also altered in rho zero cells, with circular formations as seen in Figs. 1B and C common in rho zero cells in contrast to parallel lines of cristal membrane extending across the width of the wild type mitochondrial profile as seen in Fig. 1A. Inner boundary membranes appear totally normal in rho zero cells. The depletion of cristae correlates with a loss of respiratory capacity in rho zero cells, consistent with the cristae being the primary location of the respiratory complexes.

The converse change, cristal amplification, has been produced by switching cultured human cells from glucose culture medium to galactose, a non-fermentable sugar. Oxygen consumption has been shown to increase on the galactose medium, indicating a rise in activity of the respiratory chain complexes. The ratio of cristal to inner boundary membrane increases from 1.5 for cells grown on glucose to 3.6 for cells grown on galactose. Additionally, galactose-grown cells have a denser matrix, enlarged intracristal spaces and electron dense granules in the matrix (Fig. 2).

In wild type MRC-5 cells grown on glucose, the cristal membranes appear different from the inner boundary membranes when viewed by freeze fracture (Fig. 3). Selective amplification and depletion of the cristal membrane relative to the inner boundary membrane is likely to reflect their different functions.

[1] R. W. Gilkerson et al., FEBS Lett. 474 (2000) 1. 
Figure 1. Mitochondria from wild type (A) and rho zero (B and C) MRC-5 human lung fibroblasts. Arrows indicate circular cristal membrane formations.

Figure 2. Mitochondria from cultured human cells. A and B are 143 B cells; C and D are Hela cells. $\mathrm{A}$ and $\mathrm{C}$ were grown on glucose and $\mathrm{B}$ and $\mathrm{D}$ were grown on galactose.

Figure 3. Freeze fracture of a mitochondrion in a wild type MRC-5 cell. The inner boundary membrane has smaller particles with slightly more space between particles. The cristal membrane is darkest because it was at the steepest angle relative to the platinum-carbon source.

All scale bars $0.2 \mu \mathrm{m}$.

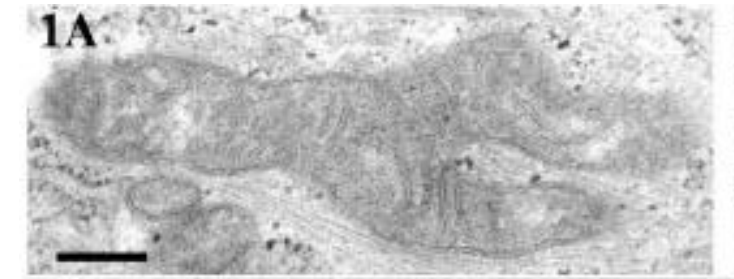

2A
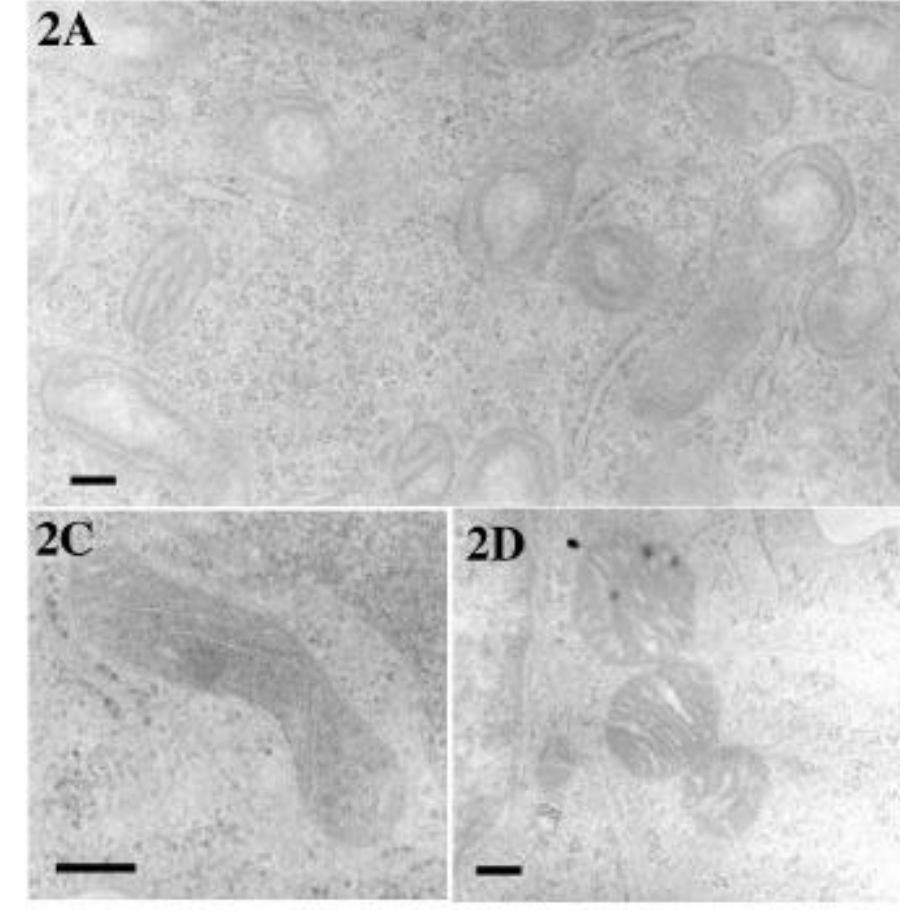
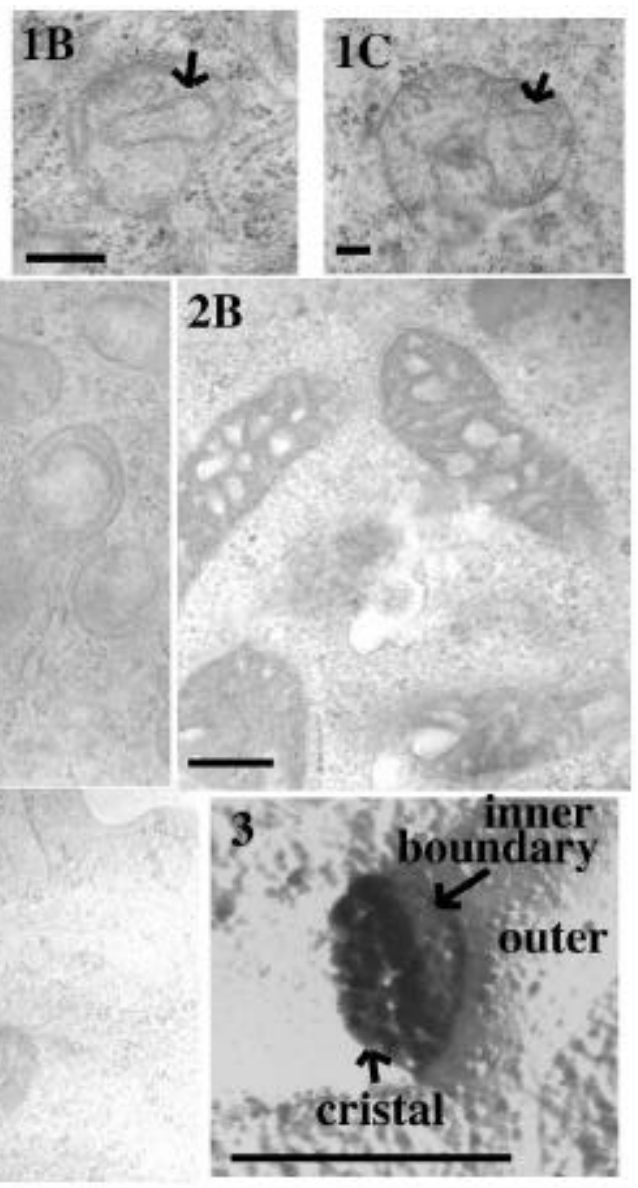